'Sección Geriatría, Servicio de Medicina Interna, Hospital Naval A. Nef, Viña del Mar, Chile. 2Departamento de Medicina, Facultad de Medicina, Universidad Andrés Bello, Viña del Mar, Chile. ${ }^{3}$ Servicio de Medicina Interna Hospital Naval A. Nef, Viña del Mar, Chile. ${ }^{4}$ Facultad de Medicina, Universidad de Valparaíso, Valparaíso, Chile.

Fuente de apoyo financiero: No presenta.

Recibido el 23 de septiembre de 2014, aceptado el 22 de enero de 2015.

Correspondencia a: Dra. Paola Fuentes R. Subida Alessandri s/n, Hospital Naval A. Nef, Viña del Mar, Chile. Teléfono: +56322573635 paolafuentesr@gmail.com

\section{Enfermedad por depósito de cristales de pirofosfato de calcio como ejemplo de forma de manifestación de la enfermedad en el adulto mayor}

\author{
PAOLA FUENTES R. ${ }^{1,2,4}$, JAVIER WEBAR ${ }^{2}$, CARLA MATUS $^{2}$, \\ CRISTIÁN VERGARA ${ }^{3}$, MICHELLE HERRERA ${ }^{4}$, \\ ELIZABETH BARTHEL M. ${ }^{2,3}$, JORGE VEGA S. ${ }^{3,4}$
}

\section{Calcium pyrophosphate dihydrate crystal deposition disease as an example of illness presentation in the elderly}

Illness presentation in the elderly may be entirely non-specific, with fatigue, loss of function or the presence of geriatric syndromes. We report a 90 years old male consulting in the emergency room for delirium that persisted throughout hospitalization without finding a cause. During the course of hospitalization mild fever appeared and a left knee swelling became apparent. A synovial fluid aspiration showed a leukocyte count of 360 per field with $60 \%$ polymorphonuclear cells. The culture was negative. With a presumptive diagnosis of pseudogout, cochicine and celecoxib were started with remission of the confusional state. The patient was discharged 13 days after admission in good conditions.

(Rev Med Chile 2015; 143: 536-539)

Key words: Aged; Chondrocalcinosis; Delirium; Fever.
$\mathrm{E}$ 1 envejecimiento de la población hará que cada día sea más frecuente para el médico el enfrentarse a adultos mayores. Estos pacientes requieren de una aproximación diagnóstica y terapéutica diferente a los adultos más jóvenes. Antiguamente se describía a las formas de presentación de las enfermedades comunes en adultos mayores como "atípicas", sin embargo, hoy se reconoce que son la forma habitual de presentación ${ }^{1-3}$. A menudo las enfermedades siguen un curso silente o lo hacen en forma larvada, otras veces originan sólo compromiso del estado general, quiebre funcional o el desarrollo de algún síndrome geriátrico (delirium, incontinencia de esfínteres, inestabilidad de la marcha, caídas, malnutrición, depresión, demencia, etc. $)^{4,5}$. Estos síndromes se ven agravados por la presencia de múltiples enfermedades crónicas concomitantes y la polifarmacia a que a menudo son sometidos estos pacientes.

El objetivo de la comunicación de este caso clínico de un paciente adulto mayor es enfatizar los conceptos previamente señalados.

\section{Caso clínico}

Paciente de 90 años con antecedentes de enfermedad diverticular del colon, reflujo gastroesofágico y déficit sensorial visual y auditivo. Usuario de eszopiclona en forma habitual. En la valoración geriátrica integral realizada al ingreso al hospital se lo describió como autovalente en actividades avanzadas de la vida diaria y sin deterioro cognitivo.

Consultó en Servicio de Urgencia por presentar en las últimas 24 h cefalea holocránea intensa, 
desorientación témporo-espacial y agitación psicomotora de inicio brusco. Sus familiares no refirieron la presencia de fiebre, náuseas o vómitos. En el examen físico destacó la presencia de taquicardia regular, hipertensión arterial y ausencia de focalización neurológica. La tomografía computada de cerebro mostró involución encefálica y ateromatosis en el sistema vertebro-basilar. No había signos de infartos ni hemorragias. Los exámenes de laboratorio no evidenciaron alteraciones metabólicas ni hidroelectrolíticas y la proteína $\mathrm{C}$ reactiva estaba en concentración normal. Fue evaluado por neurólogo quien describió la presencia de delirium y una probable hemianopsia homónima izquierda, sospechando un accidente cerebrovascular isquémico derecho. Al día siguiente se describe que el paciente no presentaba delirium, en un nuevo examen neurológico se descartó la hemianopsia. Durante el día presentó febrículas hasta $37,5^{\circ} \mathrm{C}$. Al cuarto día de hospitalización presentó nuevamente delirium. Una tomografía computada de cerebro de control no mostró cambios; sin embargo, la proteína $\mathrm{C}$ reactiva se elevó $(125 \mathrm{mg} / \mathrm{L})$ y apareció discreta leucocitosis. Al día siguiente apareció fiebre de $38^{\circ} \mathrm{C}$. Se sospechó un cuadro infeccioso efectuándose una radiografía de tórax, examen de orina, urocultivo, hemocultivos y obtención de líquido cefalorraquídeo mediante una punción lumbar, los que resultaron normales o negativos. Una evaluación por infectólogo sugirió emplear terapia antibiótica empírica, prescribiéndose ceftriaxona y ampicilina. La persistencia del delirium y de la elevación de los parámetros inflamatorios al cuarto día de terapia antibiótica motivó la realización de una tomografía computada de tórax, abdomen y pelvis y de una ecotomografía abdominal, las que sólo mostraron colelitiasis sin signos de colecistitis aguda. Dos días más tarde el paciente presentó aumento de volumen en rodilla izquierda con signos inflamatorios. La punción articular obtuvo un líquido amarillo, levemente turbio, con viscosidad $<0,1$. El recuento celular fue de 360 leucocitos por campo, con $60 \%$ de polimorfonucleares. El gram y cultivo bacteriano resultaron negativos. La radiografía de la rodilla mostró calcificación del cartílago articular compatible con condrocalcinosis (Figura 1). Se concluyó que el síndrome febril, elevación de parámetros inflamatorios, delirium y monoartritis de rodilla con las características del líquido

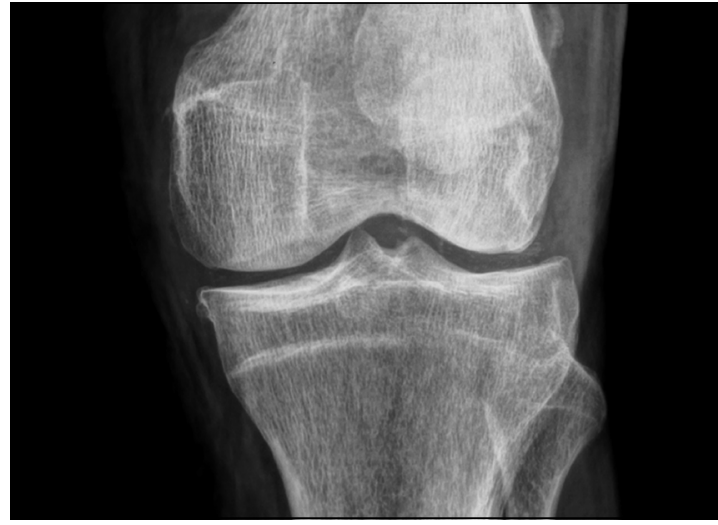

Figura 1. Radiografía Anteroposterior de Rodilla Izquierda.

articular descritas fueron ocasionados por una crisis de pseudogota. No se efectuó un análisis de líquido articular para búsqueda de cristales por no contar el laboratorio de urgencias del hospital con este método. Se suspendieron los antibióticos y se inició terapia con celecoxib y colchicina. La fiebre, el delirium y la artritis remitieron en $48 \mathrm{~h}$. El paciente egresó a los 13 días de hospitalización en buen estado general y en los controles posteriores estaba en la condición que tenía previo a la enfermedad que motivó la hospitalización.

\section{Discusión}

Este caso muestra que la forma de presentación de una enfermedad común en un adulto mayor es diferente a la de un adulto más joven. Este paciente, a pesar de tener un buen nivel cognitivo y funcional de base, debutó con delirium y síndrome febril como primera manifestación de una crisis de pseudogota.

La fiebre es un signo clásico de infección. Esta puede estar ausente o presentarse tardíamente en los adultos mayores, debido a su dificultad en montar una respuesta inmune efectiva ${ }^{6}$. Las causas más frecuentes de fiebre originada en infecciones en el adulto mayor corresponden a las localizaciones respiratorias y urinarias ${ }^{1}$. Cuando no se origina en infecciones, las causas más frecuentes son las patologías reumatológicas ${ }^{7}$. La fiebre en los pacientes con enfermedad por depósito de cristales de pirofosfato de calcio se atribuye a la interleuquina 1 producida por monocitos estimulados 
por los cristales. Esta misma interleuquina puede afectar el sistema nervioso central produciendo alteración de ritmos circadianos ${ }^{8}$.

La enfermedad por depósito de cristales de pirofosfato de calcio es una enfermedad heterogénea que se caracteriza por la presencia de cristales intraarticulares. Tiene tres presentaciones clásicas que consisten en sinovitis aguda (llamada también pseudogota, como en este caso), artritis crónica y condrocalcinosis asintomática ${ }^{9}$. La pseudogota es una causa poco común de fiebre y elevación de parámetros inflamatorios, sin embargo, es la causa más común de monoartritis en el adulto mayor9. El término condrocalcinosis se utiliza para denominar a la calcificación del cartílago hialino y/o fibrocartílago evidenciada mediante una radiografía convencional ${ }^{10-12}$. El depósito de cristales se produce principalmente en el cartílago pero también puede producirse en el espacio articular. El sitio más común de compromiso es la rodilla, seguido de la muñeca y articulación glenohumeral ${ }^{10}$. Esta enfermedad tiene una estrecha relación con la artrosis, por lo que afecta principalmente a adultos mayores, pero puede ocurrir en pacientes más jóvenes que tienen una variante familiar, como la familia descrita en 1975 en nuestro país ${ }^{13}$.

Su prevalencia en mayores de 60 años se ha estimado en $10 \%{ }^{14}$. Como la prevalencia aumenta a medida que aumenta la edad, esta patología debe sospecharse en adultos mayores con monoartritis aguda ${ }^{15}$. Como lo mostró este caso, su presentación en adultos mayores puede ser diferente a la presentación en adultos más jóvenes ${ }^{14}$.

Los criterios diagnósticos de enfermedad por depósito de cristales de pirofosfato de calcio de McCarthy consisten en la demostración de cristales de pirofosfato de calcio en tejidos o líquido sinovial, documentando la presencia de cristales con birrefringencia positiva débil por microscopia óptica con luz polarizada y los hallazgos radiográficos de calcificación en cartílago o unión articular. La presencia de ambos se considera enfermedad definitiva y de uno de los dos criterios como probable ${ }^{16}$. Debido a que la birrefringencia de los cristales es débil, muchos laboratorios pueden no detectarlos, por lo que se enfatiza la importancia de un observador experimentado en el análisis del líquido y detección de cristales $^{17}$, como sucedió en el presente caso en que el líquido sinovial, por el horario de la pun- ción, no fue analizado por experto; sin embargo, el cumplir con los otros criterios, hizo plantear el diagnóstico como probable, a lo que se agrega haber descartado otras patologías posibles, como la infecciosa, y la excelente respuesta al tratamiento. Dentro de los diagnósticos diferenciales se incluye la artritis séptica ${ }^{17}$ que se descartó por las características macroscópicas del líquido articular, bajo recuento celular del líquido, cultivo de líquido articular negativo y buena respuesta del paciente a terapia antiinflamatoria, con retiro precoz de antibióticos.

El delirium es uno de los síndromes geriátricos más frecuentes. Su inicio es agudo, tiene un curso fluctuante y su origen es multifactorial ${ }^{18}$. En geriatría es considerado como una emergencia médica $^{18,19}$. Cuando se presenta debe buscarse su etiología, la que no siempre es fácilmente identificable ${ }^{20}$, como lo fue este caso. Se debe hacer un balance entre los factores predisponentes (en este caso sólo la edad y uso de fármacos potencialmente inapropiados como la eszopiclona) y precipitantes, teniendo en cuenta que un paciente con pocos factores predisponentes requiere una injuria mayor para que se desencadene el delirium ${ }^{19}$. El diagnóstico diferencial de delirium en el adulto mayor incluye un espectro amplio de patologías de múltiples sistemas. Las causas más frecuentes son las infecciosas (respiratorias, urinarias y cutáneas), uso de fármacos potencialmente inapropiados como los anticolinérgicos, alteraciones metabólicas e hidroelectrolíticas y otras menos frecuentes son las cardiovasculares y neurológicas. Se mencionan en la literatura como enfermedades reumatológicas precipitantes de delirium la pseudogota, artritis reumatoidea y arteritis de la temporal ${ }^{1}$. En la literatura encontramos dos reportes previos de delirium y pseudogota ${ }^{8,21}$.

El delirium se asocia a mayor morbilidad, hospitalizaciones prolongadas, aumento de los costos en salud, deterioro funcional, institucionalización y mortalidad ${ }^{18,19,20}$. El delirium además es una experiencia traumática tanto para el paciente como para su familia ${ }^{22}$.

En suma, este caso ejemplifica que la forma de presentación de una enfermedad común en un adulto mayor es diferente a la presentación clásica en un adulto más joven. La fiebre y el delirium comúnmente están asociados a infecciones, pero pueden asociarse a cuadros inflamatorios no infecciosos como lo fue en este caso. 


\section{Referencias}

1. Rosen S, Koretz B, Reuben D. Presentation of Disease in Old Age. En: Brocklehurst's Textbook of Geriatric Medicine and Gerontology. Fillit HM et al, 7th Ed 2000, pages 205-210. ISBN 01416062319.

2. Boss GR, Seegmiller JE. Age-related physiological changes and their clinical significance. West J Med 1981; 135 (6): 434-40.

3. García Ocaña R, Ayuso Moreno R, Arranz de la Cerda A, Colomo Rodríguez M, Robles Agudo F. Presentación atípica de la enfermedad en el anciano. Un caso de fiebre de origen desconocido. Rev Esp Geriatr Gerontol 2001; 36 (6): 361-4.

4. Hodkinson HM. Non-specific presentation of illness. Br Med J 1973; 4 (5884): 94-6.

5. Schwab WS. Geriatric syndromes. J Am Geriatr Soc 2008; 56 (2): 363-4.

6. Fernández Moyano A, Mata Martín AM, Espinosa Calleja R. Febrile syndrome in an elderly patient. Rev Clin Esp 2008; 208 (11): 564-7.

7. Cagatay AA, Tufan F, Hindilerden F, Aydin S, Elcioglu OC, Karadeniz A, et al. The Causes of Acute Fever Requiring Hospitalization in Geriatric Patients: Comparison of Infectious and Noninfectious Etiology. J Aging Res 2010; 12: 380892.

8. Aragón A, Sepúlveda MA, Cerón A. Confusional syndrome as the presenting form of calcium pyrophosphate dihydrate deposition disease. Br J Rheumatol 1992; 31 (2): 144. Pub Med PMID: 1737232.

9. Song JS, Lee YH, Kim SS, Park W. A case of calcium pyrophosphate dihydrate crystal deposition disease presenting as an acute polyarthritis. J Korean Med Sci. 2002; 17 (3): 423-5.

10. Rosenthal AK, Terkeltaub RA. Calcium Pyrophosphate Dihydrate (CPPD) Crystal Deposition Disease. A Clinician's Pearls and Myths in Rheumatology. Springer London, 2010. 369-76.

11. Ea HK, Lioté F. Calcium pyrophosphate dihydrate and basic calcium phosphate crystal-induced arthropathies: update on pathogenesis, clinical features, and therapy. Curr Rheumatol Rep 2004; 6 (3): 221-7.

12. Zhang W, Doherty M, Bardin T, Barksova V, Guerne PA, Jansen TL, et al. European League Against Rheumatism recommendations for calcium pyrophosphate deposition. Part I: terminology and diagnosis. Ann Rheum Dis 2011; 70 (4): 563-70.

13. Reginato AJ, Hollander JL, Martínez V, Valenzuela F, Schiapachasse V, Covarrubias E, Jacobelli S, et al. Familial chondrocalcinosis in the Chiloe Islands, Chile. Ann Rheum Dis 1975; 34 (3): 260-8.

14. Felson DT, Anderson JJ, Naimark A, Kannel W, Meenan RF. The prevalence of chondrocalcinosis in the elderly and its association with knee osteoarthritis: the Framingham Study. J Rheumatol 1989; 16: 1241-5.

15. Sack K. Monoarthritis: differential diagnosis. Am J Med 1997; 102 (1A): 30S-34.

16. Rosenthal AK, Ryan LM, McCarty DJ. Calcium pyrophosphate crystal deposition disease, pseudogout, and articular chondrocalcinosis. In: Arthritis and Allied Conditions, 15th ed, Koopman, WJ, Moreland, LW (Eds), Lippincott Williams \& Wilkins, Philadelphia 2005, p. 2373.

17. Wise CM. Crystal-associated arthritis in the elderly. Rheum Dis Clin North Am 2007; 33 (1): 33-55.

18. Inouye S. Delirium in Older Persons. N Engl J Med 2006; 354: 1157-65.

19. Han JH. Delirium in the older emergency department patient: a quiet epidemic. Emerg Med Clin North Am 2010; 28 (3): 611-31.

20. Marcantonio ER. In the clinic. Delirium. Ann Intern Med 2011; 154 (11).

21. Lin P, Tangchaivang N. A Case of Fever and Altered Mental Status Caused by Calcium Pyrophosphate Dehydrate (CPPD) Deposition Disease. Proceedings of Healthcare -VOLUME 16 (2012).

22. O'Malley G, Leonard M, Meagher D, O'Keeffe ST. The delirium experience: a review. J Psychosom Res 2008; 65 (3): 223-8. 